\title{
Einfache Versorgung, schwieriger Fall - Regelversorgung
}

Nicht immer ist es die „High-End-Versorgung“, welche im Normalfall durchgeführt wird. Ob aus finanziellen, allgemeinmedizinischen Gründen, oder aber auch, weil der Patient sich schlichtweg für eine „einfache“ Arbeit entscheidet. Aber die „einfachen" Versorgungen sind nicht immer die leicht durchzuführenden Behandlungen.

Für ein wirtschaftlich und prothetisch gutes Ergebnis ist es von großer Wichtigkeit eine schnelle und unkompliziert Behandlung, mit verlässlichen Materialien durchzuführen, ohne Einfluss welche Arbeit und welche Umstände bewältigt werden müssen.

\section{Patientenfall}

Multimorbider, marcumarisierter, 76-jähriger Patient mit einem erhaltungswürdigen Restzahnbestand 16, 25-27. Der Patient ist mit einer provisorischen Prothese versorgt. 25 und 26 weisen sehr kurze klinische Kronen auf. Beide Zähne sind überkronungsbedürftig. Lange Behandlungen

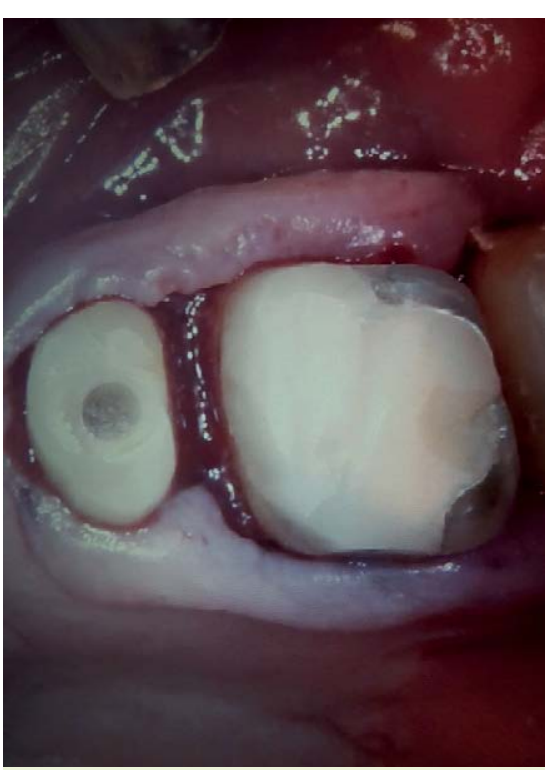

Die Präparation. sind aufgrund der allgemeinmedizinischen Situation nicht möglich. Aus allgemeinmedizinischen und finanziellen Gründen musste der Patient schnell, einfach und langfristig, wenn möglich auch erweiterbar, mit Zahnersatz im Oberkiefer versorgt werden.

\section{Prothetische Planung}

- Überkronung der Zähne 25, 26

- Modellgußprothese zum Ersatz der Zähne 15-24

\section{Erschwerte Bedingungen \\ - Marcumar wurde nicht abgesetzt \\ - Subgingivale Präparationsgrenzen müssen trotz Blutungsneigung zuver- lässig dargestellt werden.}

\section{Vorgehen}

Für eine exakte Abformung ist es notwendig ein Material zu wählen, welches mit der Tendenz zur Blutung umgehen kann und trotzdem im subgingivalen Bereich Detailtreue und Stabilität mitbringt. Außerdem sollte die Abformung schnell durchgeführt werden können, um dem Patienten eine kurze Behandlungszeit zu gewährleisten. Wir haben uns für eine Doppelmischabformung mit dem Vinylsiloxanether $^{\circledR}$ „Identium ${ }^{\circledR}$ heavy und light" der Firma Kettenbach entschieden (oAbb. 1, 2).

Nach Anwendung von Expasyl ${ }^{\circledR}$, gründlicher Reinigung und Trocknung, wird der Abformlöffel mit dem fließfähigen und

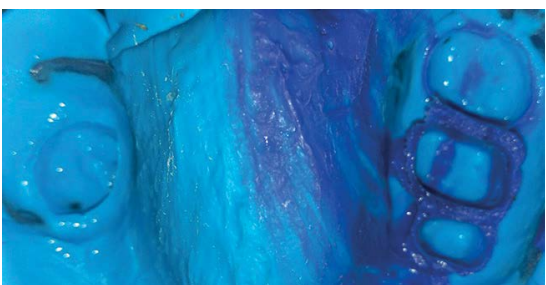

Abformung der Präparation.

doch standfesten Trägermaterial Identium heavy beschickt. Während dessen erfolgt die Umspritzung der Stümpfe mit Identium light unter gleichzeitiger Absaugung. Das Identium light hat eine sehr schöne initiale Hydrophilie und zieht sich fast zusehends in den Sulcus der Zähne, obwohl dieser immer noch leicht mit Sulcusfluid und Blut gefüllt ist. Die Entnahme ist auch für den Patienten annehmbar.

Auch den Geschmack beurteilt der Patient eher gut. Trotz relativ starker Blutungsneigung des Patienten und auch deutlicher Sulcusblutung kann auch im subgingivalen Bereich eine detailgenaue und unterbrechungsfreie Präparationsgrenze dargestellt werden. Trägermaterial und Korrekturmaterial sind gut und ohne Übergänge miteinander verbunden.

Nach Fertigstellung der Kronen 25 und 26 wurde, über die Kronen, eine individuelle Abformung vorgenommen. Hierzu wurde nochmals Identium heavy genutzt. Auch hier erhalten wir eine detailgenaue Abformung auf deren Grundlage die Modellgußprothese hergestellt werden konnte.

\section{Fazit}

$\nabla$

Aufgrund des guten Verhaltens von Identium konnte, trotz moderater Blutung, zuverlässig und schnell abgeformt werden. Als Doppelmisch-, wie auch als Einphasenmaterial hat Identium durch seine positiven Eigenschaften überzeugt.

\section{Kontakt}

Dr. Stephanie Etzbach

ZAP Dr. Hilmar von Schwertführer 46325 Borken

Dieser Beitrag entstand in Zusammenarbeit mit der

Kettenbach GmbH \& Co. KG, Eschenburg. 